\title{
HEMATOMA SUBCAPSULAR HEPATICO POR FASCIOLASIS
}

\author{
Drs. Humberto Rosas $L^{(1)}$, Carlos Fiestas $F^{(2)}$, Richard Torres $M^{(2)}$, Giorgio Aita $C^{(2)}$, David Lozada $\mathbf{M}^{(2)}$.
}

1. Jefe del Servicio de Imaginología del Hospital Nacional "Almanzor Aguinaga Asenjo", Chiclayo Perú.

2. Médico Residente del Hospital Nacional Almanzor Aguinaga Asenjo, Perú.

\section{HEPATIC SUBCAPSULAR HEMATOMA CAUSED BY FASCIOLIASIS}

\begin{abstract}
Abtract: Hepatic fasciolasis is a worldwide spread zoonoses mainly affecting cattle-raising countries. It is caused by the trematode Fasciola hepatica and it is characterized by abdominal pain, fever, nausea and vomitus, weight loss, diahrrea, paleness, general malaise, and hypereosinophilia. Immunological diagnosis as well as stool eggs count may be performed. Hepatic subcapsular and intraparenchymatous hematoma is an infrequent complication of human fascioliasis. Nevertheless, for establishing a proper diagnosis and treatment, any suspicion of its presence must be carefully discarded through, clinical epidemiology, laboratory and imaging exams and procedures. The aim of this study is to expand knowledge on this unfrequently dealt pathology in medical literature by presenting four case reports related to patients undergoing a two-year treatment. All of them had been referred from Departamento de Cajamarca, Peru.
\end{abstract}

Key words: Fasciolasis, Hepatic Hematoma, Zoonoses.

Resumen: La fasciolasis hepática es una zoonosis mundialmente difundida, sobre todo en los países productores de ganado; causada por la fasciola hepática. Se manifiesta por dolor abdominal, fiebre, náuseas y vómitos, baja de peso, diarrea, palidez, malestar general e hipereosinofilia. El diagnóstico es inmunológico y también puede hacerse por recuento de huevos en heces.

El hematoma subcapsular e intraparenquimatoso hepático es una complicación rara de la fasciolasis humana pero se debe tener un alto índice de sospecha uniendo epidemiología, clínica, laboratorio e imagino-

Rosas H. y cols. Hematoma subcapsular hepatico por fasciolasis. Rev Chil Radiol 2007; 14: 20-24.

Correspondencia: Dr. Humberto Rosas $L$. betoimagen2@hotmail.com logía para un adecuado diagnóstico y tratamiento. El propósito de reportar estos casos es dar a conocer una patología poco frecuente en la literatura, con una casuística de cuatro pacientes tratados en el lapso de dos años, todos referidos del Departamento de Cajamarca.

Palabras clave: Fasciolasis, Hematoma hepático, Zoonosis.

\section{Introducción}

La fasciolasis hepática $(\mathrm{FH})$ es una enfermedad tropical reemergente, endémica en América Latina $^{(1,2)}$. El agente es la fasciola hepática, un tremátodo hermafrodita plano no segmentado, que mide $2-3 \mathrm{~cm}$ de longitud por 10-13 $\mathrm{mm}$ de ancho ${ }^{(3)}$. Tiene dos ciclos:

1. Sexual: que ocurre en el huésped definitivo (rumiantes, otros animales y el hombre).

2. Asexual: que se desarrolla en el huésped intermediario (moluscos del género fossarea y lymnaea) $)^{(4,5)}$.

La FH es un problema de salud pública en Perú, Bolivia, Chile y Ecuador(6); en el Perú la prevalencia es de $34 \%{ }^{(7,8)}$ y en Cajamarca entre $0.4-40 \%{ }^{(9)}$.

El cuadro clínico abarca desde la fase asintomática hasta cuadros severos de hemorragia intraabdominal y abdomen agudo quirúrgico ${ }^{(10)}$. En el período de invasión (paso del duodeno al parénquima hepático) se manifiesta por dolor mal localizado en la mitad superior del abdomen, hepatomegalia, fiebre intermitente, urticaria, etc. y en el período de estado (fasciola en vía biliar) por anorexia, flatulencia, náuseas, dolor tipo cólico biliar, ictericia y fiebre ${ }^{(2,3,10-12)}$.

El diagnóstico se realiza en la fase aguda por los métodos serológicos y en la fase crónica por el hallazgo de huevos en heces. El tratamiento es bitionol, praziquantel, triclabendazol ${ }^{(3,4,8-10,12-15)}$.

El objetivo de este trabajo es reportar el hematoma hepático como consecuencia de fasciola, ya que son pocos los reportes encontrados en la literatura. 


\section{Material y método}

Se analizan cuatro casos, que corresponden a pacientes que fueron referidos de Cajamarca. Todos requirieron hospitalización.

El estudio fue retrospectivo; los datos se obtuvieron de las historias clínicas y las imágenes del Servicio de Imaginología del Hospital Nacional "Almanzor Aguinaga Asenjo".

\section{Resultados}

Caso clínico 1. Varón de 40 años de edad, médico, procedente de Cajamarca; portador de policitemia, con antecedente de TBC a los 9 años y traumatismo abdominal 6 años atrás.

Ingresa al Hospital Almanzor Aguinaga Chiclayo en abril 2005 por presentar desde hace 15 días dolor abdominal difuso de predominio en hipocondrio derecho y epigastrio, irradiado a fosa ilíaca derecha y hombro ipsilateral, de presentación diaria y que se exacerba al movilizarse. Refiere además sensación de alza térmica, calofríos y malestar general.

PA: 110/70 mm Hg. FC: 78 lat/min. FR: 20 resp/min $\mathrm{T}: 36.6^{\circ} \mathrm{C}$.

Al examen físico, el abdomen aparece globuloso, doloroso a la palpación profunda en hipocondrio derecho.

En los exámenes de laboratorio destaca: GB: $20.500 \times \mathrm{mm}^{3}$ con $12 \%$ de eosinófilos (2460), $\mathrm{Hb}$ : $9.06 \mathrm{mg} / \mathrm{dl}$, Hto: $27 \%$, Plaq: $349.000 \mathrm{~mm}^{3}$. Fosf. Alcal: 232 U/I (N: 40-129). GGTP: 174 U/L (N: 1150). TGO: $172 \mathrm{U} / \mathrm{L}$ (N: 5-38). TGP: $212 \mathrm{U} / \mathrm{L}$ (N: 5-40). BT: $0.62 \mathrm{mg} / \mathrm{dl}$ (N: 0.3-1.0). El diagnóstico fue confirmado por arco II, Elisa y Western blot para fasciola.

En la tomografía computada (TC) abdominal con contraste (Figura 1) se observa gran colección heterogénea, predominantemente isodensa contactando los segmentos VI y VII, con densidades de 42 - $53 \mathrm{UH}$, compatible con hematoma subcapsular hepático; además se aprecian otros pequeños hematomas intrahepáticos. Una semana más tarde, nueva TC abdominal (Figura 2) muestra persistencia de gran colección en los segmentos hepáticos mencionados. Se efectuó gammagrafía con glóbulos rojos marcados (Figura 3) con administración ev de $148 \mathrm{MBq}$ de Sulfuro coloide $99 \mathrm{mTC}$, que demuestra extensa área hipocaptante que ocupa casi la totalidad del lóbulo derecho.

Dos semanas más tarde, un control de TC muestra discreta regresión del tamaño de la lesión (Figura 4). Se controló con US abdominal 6 meses más tarde, observándose imagen de ecogenicidad heterogénea algo más pequeña, con un volumen estimado de 392 cc.

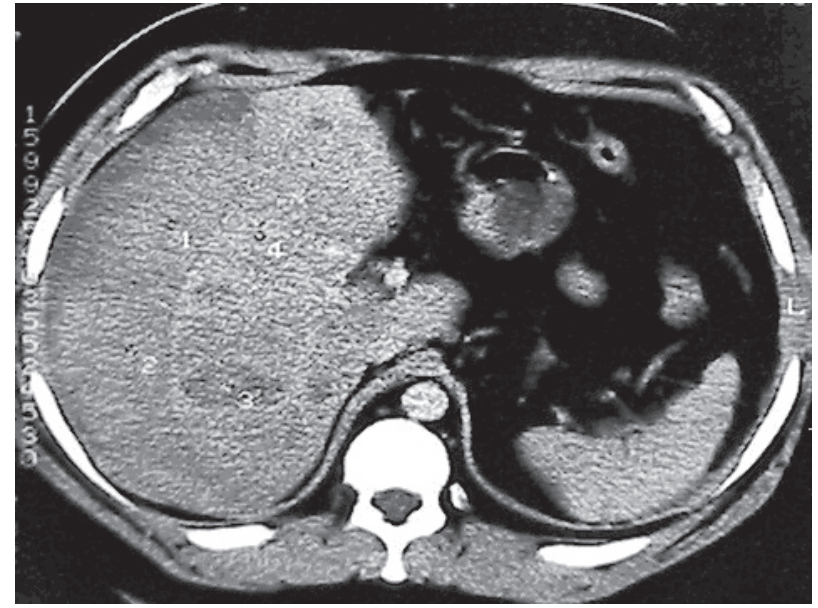

Figura 1. Caso 1. TC abdominal con contraste muestra gran colección subcapsular heterogénea, con densidades de 42 a 53 U.H, asociada a pequeños hematomas intrahepáticos.

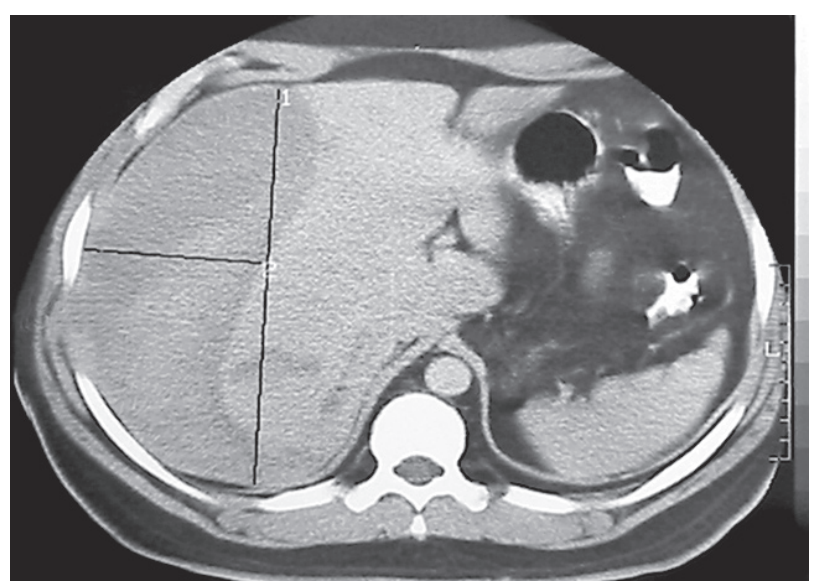

Figura 2. Caso 1. Control de TC efectuada una semana más tarde, demuestra colección hipodensa que mide 19,9 $x 8 \mathrm{~cm}$.
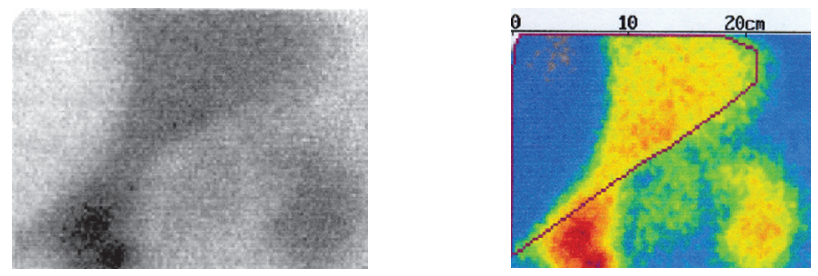

Figura 3 a, b. Caso 1. Gammagrafía hepática con glóbulos rojos marcados con sulfuro coloide 99mTC, muestra extensa área hipocaptante que ocupa casi la totalidad del lóbulo derecho.

Caso clínico 2. Varón de 59 años de edad, procedente de Chota-Cajamarca, con antecedente de hipertensión arterial. Consulta por una semana de dolor abdominal tipo cólico en el cuadrante superior derecho de moderada intensidad, tratado ambulatoriamente con analgésicos y antiespasmódicos con 
persistencia de la sintomatología e incremento de la intensidad del dolor. Concomitantemente presenta fiebre y náuseas. PA: 110/60 mm Hg. FC: 88 lat $/ \mathrm{min}$, FR: $18 \mathrm{resp} / \mathrm{min}, \mathrm{T:} 38.6^{\circ} \mathrm{C}$.

Al examen físico, el abdomen está blando, depresible, con dolor leve a palpación profunda en el cuadrante superior derecho.

Laboratorio: GB: $15.900 \mathrm{~mm}^{3}$ con $49 \%$ de eosinófilos (7.791), Hb: 12.5 mg/dl, Hto: 37\%, Plaq: 439. $000 \times \mathrm{mm}^{3}$.

BT: 25.7 umol/L (N: hasta 17.1) BD: $2.7 \mathrm{umol} / \mathrm{L}$ (N: hasta 3.42) Bl: 23.0 umol/L (N: hasta 13.7)

Se efectuó TC abdominal con contraste (Figura 5) que demuestra colección hipodensa, de bordes bien definidos, lisos, de 16,6 x 4,2 cm, adyacente a los segmentos VI y VII, compatible con hematoma subcapsular. Un control de TC abdominal demuestra persitencia de colección hipodensa, de bordes lisos, cuya densidad oscila entre 18.5 hasta $43.3 \mathrm{UH}$.

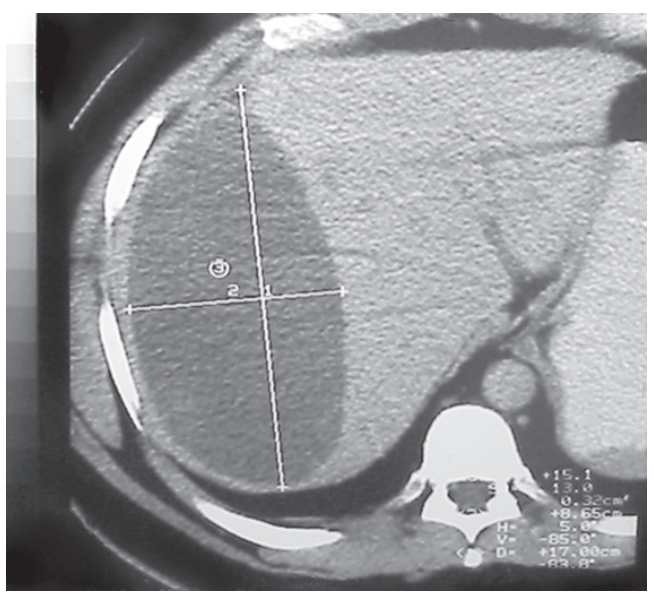

Figura 4. Caso 1. TC abdominal dos semanas más tarde, muestra persistencia de colección hipodensa de 4.2 UH, que mide $17 \times 13 \times 10 \mathrm{~cm}$.

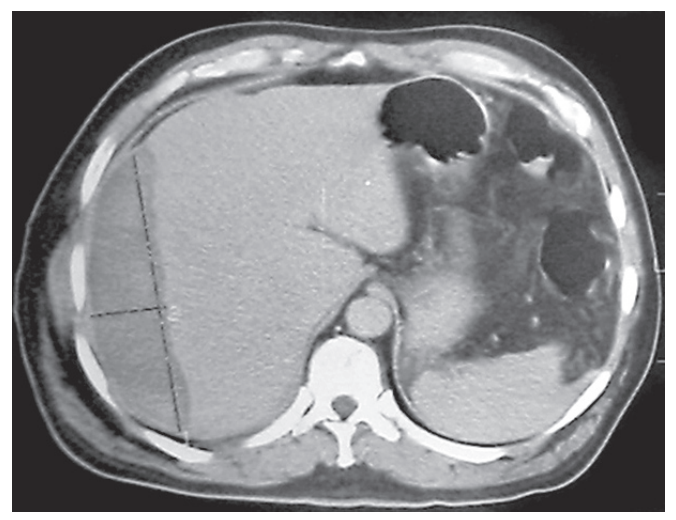

Figura 5. Caso 2. TC abdominal con contraste evidencia colección hepática hipodensa, de bordes bien definidos, que mide 16,6 6 4,2 cm, compatible con hematoma subcapsular.

Caso clínico 3. Mujer de 76 años, procedente de Jaén, con antecedente de colecistectomía y anastomosis biliodigestiva 7 años atrás.

Ingresa a nuestro hospital por presentar dolor abdominal en hipocondrio derecho desde hace 15 días, asociado a náuseas, vómitos, ictericia y fiebre persistente.

Al examen: PA: 130/80 mm Hg, FC: 76 lat/min, FR: $21 \mathrm{resp} / \mathrm{min}, \mathrm{T}: 37.9^{\circ} \mathrm{C}$.

Al examen físico destacan escleras ictéricas y dolor a la palpación profunda en hipocondrio derecho.

Laboratotorio: GB: $7.000 \times \mathrm{mm}^{3}$ con $0 \%$ eosinófilos, $\mathrm{Hb}: 10.3 \mathrm{mg} / \mathrm{dl}$, Hto: $27 \%$, Plaq: $384.000 \mathrm{x}$ $\mathrm{mm}^{3}$. Fosf. alcal: $220 \mathrm{U} / \mathrm{l}$ (N: 40-129). GGTP: 328 $\mathrm{U} / \mathrm{L}(\mathrm{N}: 8-45)$,

TGO: 48 U/L (N: 0-31), TGP: $45 \mathrm{U} / \mathrm{L}$ (N: 0-38), BT: 27.7 (N: Hasta 7.1 umol/L), BD: 12.5 (N: Hasta $3.4 \mathrm{umol} / \mathrm{L}), \mathrm{BT}: 15.2$ (N: Hasta 13.7umol/L).

La TC Abdominal con contraste (Figura 6) muestra dos imágenes hipodensas en segmentos VI y VIII, la mayor de $13,3 \times 7,1 \mathrm{~cm}$ y otra de $8,1 \times 5,1 \mathrm{~cm}$, con densidades de 24-46 UH. El diagnóstico fue confirmado por arco II, Elisa y Western blot para fasciola.
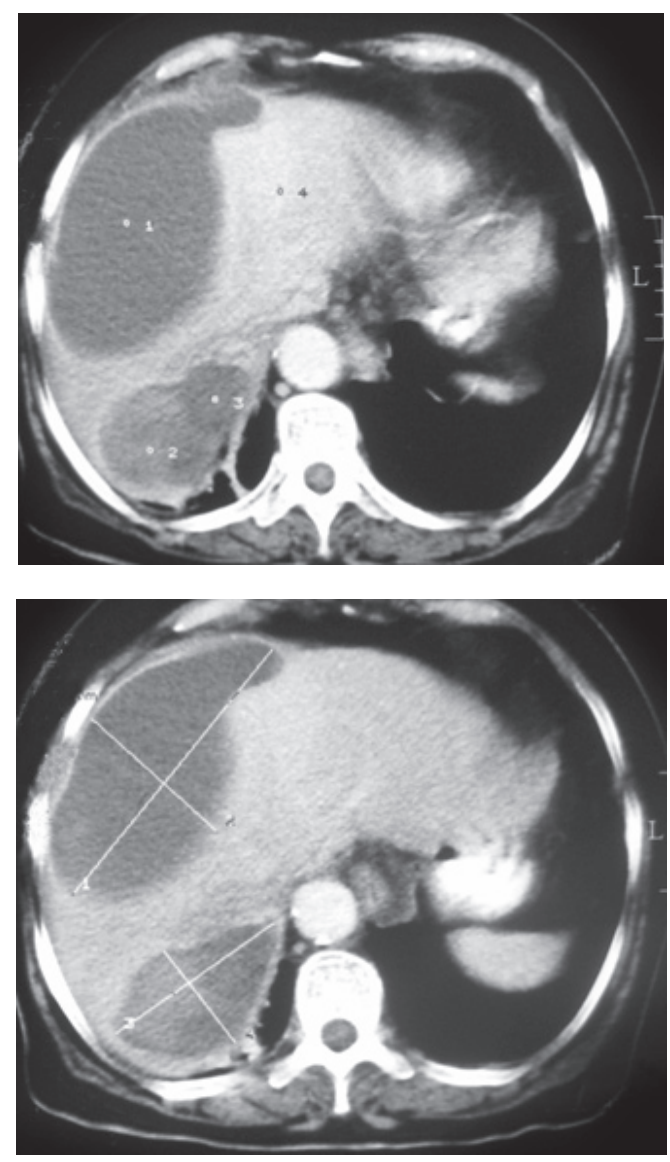

Figura 6. Caso 3. TC abdominal evidencia dos imágenes hipodensas hepáticas, la mayor de $13,3 \times 7,1 \mathrm{~cm}$ y la otra de $8,1 \times 5,1 \mathrm{~cm}$, con densidades de 24 a $46 \mathrm{UH}$.

Caso clínico 4. Mujer de 47 años de edad, procedente de Cajamarca, con antecedente de asma, hipertensión arterial, migraña y cirugías de quiste ovárico y prolapso.

Ingresa al hospital por presentar desde hace 10 días dolor abdominal en epigastrio, con irradiación a 
hipocondrio derecho, además de náuseas, vómitos, y diarrea amarillenta 7 a 8 veces al día.

Al examen, PA: 130/80 mm Hg. FC: 75 lat/min FR: $18 \mathrm{resp} / \mathrm{min} \mathrm{T}: 37.2^{\circ} \mathrm{C}$.

Regular estado general, pálida. Al examen abdominal se observa dolor a la palpación profunda en hipocondrio derecho.

En el laboratorio: GB: $9.370 \times \mathrm{mm}^{3}$ con $18 \%$ de eosinófilos, Hb: 9.9mg/dl, Hto: 27\%, Plaq: 342.000 mm³.

La TC Abdominal con contraste (Figura 7) mostró lesiones en relación a segmentos VI, VII y VIII, la superior hipodensa $(6 \mathrm{UH})$ y la inferior hiperdensa ( $25 \mathrm{UH}$ ), que en conjunto miden $16,3 \times 15,4 \times 7,8 \mathrm{~cm}$, (1.018 cc aprox.), que corresponden a hematoma subcapsular subcrónico y agudo respectivamente. Además se observa otro hematoma intraparenquimatoso, en el segmento VIII de $3 \times 2 \mathrm{~cm}$.

Se efectuó control con US abdominal dos semanas más tarde (Figura 8) que muestra imagen hipoecogénica con bandas ecogénicas, de tamaño semejante a lo observado en TC previa.

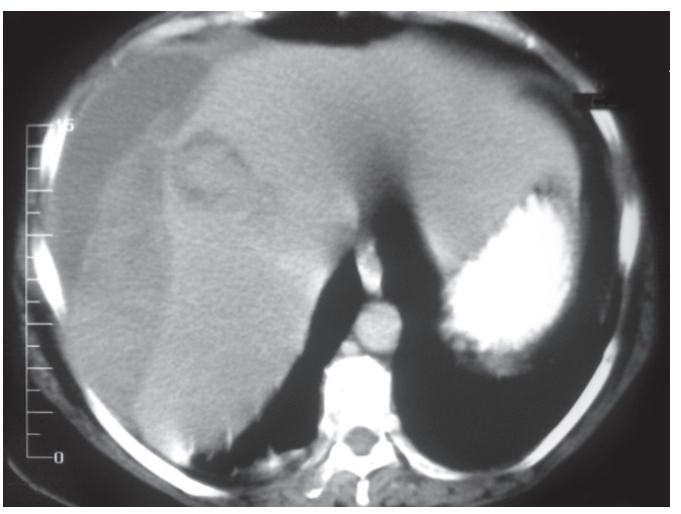

Figura 7. Caso 4. TC abdominal con contraste evidencia lesiones hepáticas con distinta densidad, que en conjunto presentan un volumen estimado de $1.018 \mathrm{cc}$, una hipodensa anterior (6 UH.) y otra hiperdensa posterior (25 UH), en relación con hematoma subcapsular subcrónico y agudo respectivamente. Existe además otro hematoma intraparenquimatoso en el segmento VIII de $3,0 \times 2,0 \mathrm{~cm}$.

En este caso, el arco II resultó en valores límites, pero por los criterios clínicos, epidemiológicos e imaginológicos se indicó tratamiento médico, con buena evolución de la paciente.

Los casos descritos proceden de zonas ganaderas. Debemos hacer notar que inicialmente la serología fue negativa en los dos primeros casos pero dada la alta sospecha, se repitieron los exámenes y en una segunda oportunidad el resultado fue positivo para fasciola en arco II y Elisa. En el tercer caso, el resultado fue contundente para fasciolasis y en el cuarto paciente el resultado obtenido no se definía entre positivo y negativo.

En todos los casos el tratamiento fue con Triclabendazol, obteniéndose buenos resultados.
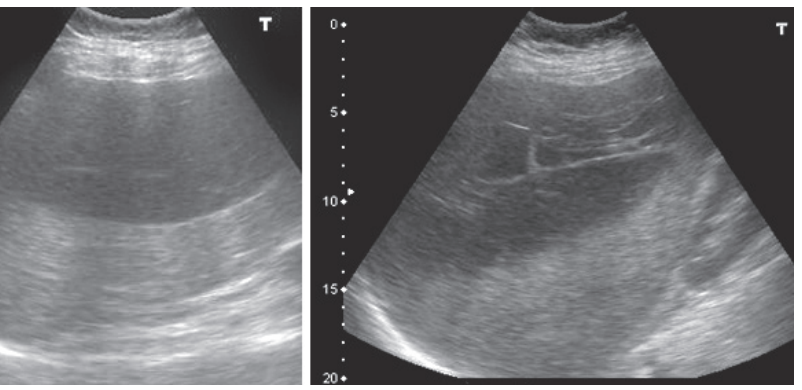

Figura 8. Caso 4. En US abdominal efectuado dos semanas más tarde, se observa imagen hipoecogénica con bandas hiperecogénicas, que mide $16 \times 15 \times 8 \mathrm{~cm}$.

\section{Discusión}

En el mundo existen aproximadamente 2.4 a 17 millones de humanos infectados por fasciola hepática ${ }^{(7,8)}$ y este número se está incrementando, sobre todo en los países en vías de desarrollo, como por ejemplo Bolivia (65-92\%), Ecuador (24-53\%) y Perú (34\%). Dos de los casos presentados provenían de Cajamarca, una de las ciudades del Perú de alta prevalencia para esta zoonosis, que se encuentra entre $0.4-40 \%(4,7,8,9)$.

Las infecciones ocurren en mayor porcentaje en varones, como se observa en nuestros casos; según Tolan y Lozano esto sería por riesgo ocupacional, dietético o recreacional ${ }^{(16,17)}$ contrario a lo reportado por Albán ${ }^{(4)}$.

La vía de contagio es la ingesta de verduras contaminadas (berros, hojas de diente de león, menta, alfalfa, junco y algunas cianofitas) y agua sin hervir $(2,4,18)$. Las formas de presentación son: aguda $(43.5 \%)$ y crónica $(55 \%)^{(4)}$.

La serie más grande en nuestro país corresponde al trabajo presentado por Gilberto Blancas, con 277 casos en un lapso de 30 años $^{(10)}$, muestra:

1. Fase aguda: dolor abdominal (91.9\%), pérdida de peso $(48.6 \%)$, náuseas y vómitos (43.2\%), hiporexia $(40.5 \%)$ y sensación de alza térmica (37.8\%). La fiebre es uno de los síntomas característicos de esta fase.

2. Fase Crónica: Dolor abdominal $(91.8 \%)$, diarrea (40.9\%) y pérdida de peso (35.9\%).

Otros autores como Picoaga, Alban, Vilca, Cornejo, Lopera reportan dolor abdominal en más del $90 \%$.

Para el diagnóstico, la presencia de hipereosinofilia (92\% de los casos) en asociación con la clínica y la epidemiología, nos permite sospechar esta patología ${ }^{(2)}$ pero, si un paciente no presenta eosinofilia no se debe descartar este diagnóstico ${ }^{(10)}$.

Las técnicas inmunológicas como arco II, Elisa y Western blot se utilizan para la fase aguda y, el recuento de huevos en heces (técnica de sedimentación rápida modificada por Lumbreras) para la fase crónica ${ }^{(8,10)}$.

En las imágenes, esta patología se presenta de dos formas: hepática y biliar. 
Al US, en FH se puede observar lesiones focales hipoecoicas, bien definidas, anfractuosas y de tamaño variable que suelen corresponder al trayecto seguido por las larvas; estas lesiones tienden a confluir en casi todos los casos. También se pueden observar adenopatías periportales. Los hematomas subcapsulares se ven como lesiones hipoecogénicas o complejas, por la presencia de coágulos.

En la fasciolasis biliar se observa engrosamiento parietal coledociano con dilatación moderada y también se puede observar el parásito en vesícula o vías biliares ${ }^{(2,8,10)}$.

En TC se puede presentar como áreas hipodensas nodulares de 1-10 mm, únicas o múltiples, o lesiones hipodensas que dan aspecto de "túnel" con disposición radiada y que se realzan con contraste. El hematoma subcapsular es una complicación poco frecuente, que se delimita mejor en la fase tardía ${ }^{(2,8,10)}$.

La gammagrafía muestra múltiples defectos intrahepáticos en la mitad de los casos.

En resonancia magnética (RM) Cevikol clasifica el aspecto de las lesiones ${ }^{(19)}$ como:

- Tipo 1: Iso o hipointensidad de la cápsula en T1, hiperintensidad de la cápsula en T2 y realce de la cápsula con gadolinio.

- Tipo 2: Area iso o hipointensa en T1, área hiperintensa mal definida en T2, con realce en forma de tractos tras la administración de gadolinio.

- Tipo 3: Lesión nodular hipointensa en T1, lesión nodular hiperintensa en $\mathrm{T} 2$, sin realce con gadolinio.

- Tipo 4: Lesión hipointensa en T1, hipo o hiperintensa central con halo periférico hiperintenso en T2, y realce periférico con gadolinio.

- Tipo 5: Focos hipointensos o áreas mal definidas en T1 y T2, con realce de zona hipointensa con gadolinio.

Este autor refiere que en el $10 \%$ de los casos la RM será normal y que el tipo 2 es el más frecuente $(62.1 \%)$.

El tratamiento consiste en bitionol, praziquantel, triclabendazol(3,4,8-10,12-15). La monoterapia puede desarrollar resistencia, por lo que recientemente en Australia se están utilizando combinaciones como Triclabendazol-Clorsulon, Triclabendazol-Luxabendazol, Triclabendazol-Closantel, Closantel-Clorsulon ${ }^{(8)}$.

\section{Conclusión}

Si bien el hematoma subcapsular hepático es una complicación poco frecuente de la fasciolasis humana, se debe tener un alto índice de sospecha uniendo la epidemiología, clínica, laboratorio e imaginología para un adecuado diagnóstico y tratamiento.

\section{Bibliografía}

1. Gotuzzo E. Enfermedades emergentes y re-emergentes en el Perú. Rev. Hered 2002; 13(2): 37-39.
2. López A, Silva C y Busel D. Fasciola Hepática: Reporte de un caso y revisión de la literatura. Rev Chil Radiol 2004; 10(2): 118-123.

3. Berenguer J. Gastroenterología y Hepatología 2da Edición. Edit. Mosby/Doyma Libros. Pág: 800-802.

4. Alban M, Jave J, Quispe T. Fasciolasis en Cajamarca. Rev. Gastroenterología. Perú 2002; 22: 28-32.

5. Huerto L, Hernández S y Acuña A. Fasciolasis en la Republica Oriental de Uruguay.

6. Cornejo W, Alva P, Sevilla C, Huiza A. Inmunodiagnóstico de la fasciolasis humana en la provincia de Chupaca-Junín, mediante ELISA de captura basado en cistanina. An. Fac. med. oct/dic 2003; 64(4): 252254.

7. Mas-Coma M, Esteban J, Bargues M. Epidemiología de la fasciolasis humana. Revisión y propuesta de una nueva clasificación. Bull Word Heath Org 1999; 77(4): 340-346.

8. Loja D, Alvizuri J, Vilca M, Avilés R, Sánchez M. Hematoma Hepático Subcapsular por fasciola. Rev Gastroenterología. Perú 2003; 23: 142 -148.

9. Jave J, Alban M, Sagastegui C, Soriano S. Tratamiento de la fasciola hepática humana con tridabendazol. Rev Gastroenterología Perú 1999, 19: 216-220.

10. Blancas G, Terashima A, Maguiña $C$, Vera L, Alvarez $H$, Tello R. Fasciolasis humana y compromiso gastrointestinal. Estudio de 277 pacientes en el Hospital Nacional Cayetano Heredia. 1970-2002. Rev. Gastroenterología. Perú 2004; 24: 143-157.

11. Rodes J, Guardia J. Medicina Interna 1era Edición. Edit. Masson 1998. Págs. 1873-1874.

12. Berenguer J, Bruguera M, García-Bengoechea M, Rodrigo $L$ eds. Tratamiento de las enfermedades hepáticas y biliares. Asociación Española para el estudio del hígado 2000. Pág. 217.

13. Ortiz P, Cabrera M, Jave J. Human Fasciolasis prevalence and treatment in a rural area of Peru. The infections disease Review - microbes of man, animals and the environment.2002 Vol 2: 42-46.

14. Jiménez J, Loja D, Ruiz E, Maco V, Marcos L, Avilés R. Fasciolasis hepática ¿Un problema diagnóstico? Rev. Gastroenterología Perú 2001; 21:148-152.

15. Savioli L. Nuevas posibilidades de lucha contra la fasciolasis. Bulletin of the World health organization 1999; 77(4): 300.

16. Tolan R. Fasciolasis 2002 www.emedicine.com

17. Lozano J y Pando Y. Distomatosis Hepática: Relación con saneamiento básico y algunos factores socioculturales. Tesis 144 Facultad Ciencias de la Salud - UNC. Junio 1993.

18. Marcos L, Maco V, Terashima A, Samalvides F, Gotuzzo E. Características clínicas de la infección crónica por fasciola hepática en niños. Rev. Gastroenterología Perú 2002; 22(3).

19. Cevikol C, Karaali K, Senol U, et al. Human fasciolasis: MR imaging findings of hepatic lesions. Eur Radiol 2003; 13: 141-148. 